\title{
Noninvasive Fault Monitoring of Electrical Machines by Solving the Steady-State Magnetic Inverse Problem
}

\author{
V. P. Bui, O. Chadebec, L.-L. Rouve, and J.-L. Coulomb \\ Grenoble Electrical Engineering Laboratory (G2ELAB-CNRS-UJF/INPG), ENSIEG, Grenoble 38402, France
}

\begin{abstract}
This paper proposes an original approach for detection, localization, and quantification of faults appearing in electrical machines. The used method in this work deals with the analysis of the external magnetic field of a machine. This approach is already known, but until now, classical methods only detect if a fault is present or not. Thus, we propose a new approach based on the theory of inverse problems. From magnetic measurements realized on sensors located around the machine, a numerical model of the machine is inverted to get the distribution of the flux in the air gap. Therefore, the analysis of this distribution can allow us not only to identify a faulty mode, but also to quantify its importance.
\end{abstract}

Index Terms-Electrical machines, flux leakage, integral method, inverse problem, method of moments, noninvasive diagnosis.

\section{INTRODUCTION}

$\mathbf{F}$ AULT monitoring of a rotating electrical machine using external magnetic field measurements is a topical subject of investigation. Indeed, the magnetic field leakage is a good representation of the magnetic induction flowing in the machine air gap. The analysis of this field is thus a powerful strategy for diagnosis, particularly because external sensors present an easiness of instrumentation.

Currently, this approach is carried out with only one sensor located close to the machine. The spectrum analysis of the recorded signal and the recognition of frequency components representative of known faults lead to a reliable diagnosis. However, these techniques offer only few possibilities for the localization or the determination of fault magnitudes.

This paper proposes an original approach based on the theory of inverse problems. The goal is to start from external magnetic field measurements realized on several sensors around the machine, to find the magnetic field distribution inside the machine air gap. By analyzing it, it will be possible to localize the fault and moreover to determine its magnitude. The second part of this paper presents the direct modeling of the problem. This model which has already been published in [1] will be quickly summarized. We will see how, from a known distribution of the magnetic induction in the machine air gap, it is possible to predict the magnetic field outside it, by taking into account the stator and the iron housing effect. In the third part, this model will be inverted. From the magnetic external induction, the distribution of the flux in the air gap will be reconstructed. Thus, it is possible to get a reliable diagnosis allowing the quantification of the fault magnitude. In the fourth part, a numerical example of short circuit identification will be presented. First experimental validations will be shown in the last part of the paper and will only focus on the direct approach.

\section{FORWARD MODELING}

The external magnetic field computation of the electrical machines is a difficult task [2]. Indeed, it is a full 3-D problem re-

Digital Object Identifier 10.1109/TMAG.2007.916593 quiring not only the analysis of the devices but also their vicinity (meaning high CPU simulation costs) and is faced to a high ratio (say $10^{6}$ ) between the internal field and the external field (introducing well-known cancellation errors problem). A methodology was developed that allows us to compute the external magnetic field with a good accuracy. It is based on a 3-D finite element modeling coupled with a post-processing based on a volume integral equation. This approach was successfully validated by experimental measurements [3].

Unfortunately, this model is too complex to be inverted, because the relation between the sources (thus the fault) and the external field is not explicit. Thus, we developed a "lighter" integral model where this relation is explicit and can be easily inverted. Our 3-D model is based on several assumptions, in particular a linear law for the materials and a simplification of the machine geometry. It has been already largely and precisely presented in [1]; however, we describe here briefly its principle.

The external magnetic field created by the machine can be represented by three distinct distributions.

1) A distribution of normal magnetic dipoles $\mathbf{T}$ (usually called double layer potential distribution) located on the average surface of the machine air gap. These dipoles are directly linked to the magnetic induction $\mathbf{B}$ flowing in the air gap. For the geometry of a known machine, it is easy to find in the literature, analytical expression of the induction B. Many references also provide these analytical expressions for failure operation modes [4].

2) A magnetic charge distribution q (usually called single layer potential distribution) located on the external surface of the stator (stator/air interface). These charges represent the flux leakage of the machine itself. They can be calculated by the following integral equation:

$$
\frac{\mu_{1 \mathrm{r}}+1}{\mu_{1 \mathrm{r}}-1} \mathrm{q}-\frac{1}{2 \pi} \iint_{\mathrm{S}_{\text {stator }}} \mathrm{q} \frac{\mathbf{r} \cdot \mathbf{n}}{\mathrm{r}^{3}} \mathrm{dS}=2\left(\mathbf{n} \cdot \mathbb{H}_{\mathbf{0}}(\mathbf{T}, \mathbf{M})\right)
$$

where $\mu_{1 \mathrm{r}}$ is the permeability of the stator and $\mathbf{H}_{0}(\mathbf{T}, \mathbf{M})$ is the field created by other sources (i.e., distribution of normal dipoles $\mathbf{T}$ and magnetization distribution $\mathbf{M}$ of the iron housing), $\mathbf{r}$ is the vector linking a point of the stator surface to the integration point, $\mathbf{n}$ is the outgoing normal of stator surface $\mathrm{S}_{\text {stator }}$. 
3) A magnetization distribution $\mathbf{M}$ located in the iron housing. It represents the shielding effect of this one. The computation of these magnetizations can be made with a classical magnetic moment method [5]

$$
\mathbf{M}-\left(\mu_{2 \mathrm{r}}-1\right) \iiint_{\mathrm{V}} \frac{\mathbf{M} \cdot \mathbf{r}}{\mathrm{r}^{3}} \mathrm{dV}=\left(\mu_{2 \mathrm{r}}-1\right) \mathbb{H}_{\mathbf{0}}(\mathbf{T}, \mathrm{q})
$$

where $\mu_{2 \mathrm{r}}$ is the relative permeability of the iron housing, $\mathrm{V}$ is the volume of this one, and $\mathbf{H}_{0}(\mathrm{~T}, \mathrm{q})$ is the field created by distribution of normal dipoles $\mathbf{T}$ and charge distribution $\mathrm{q}$.

The air gap surface, the stator surface $\mathrm{S}_{\text {stator }}$, and the iron housing volume $\mathrm{V}$ are meshed. The external magnetic field of the machine is the sum of the field created by distribution of normal dipoles $\mathbf{T}$, charge distribution $\mathrm{q}$, and magnetization distribution $\mathbf{M}$. This relation can be written thanks to a linear system

$$
\left[\mathrm{A}_{\mathrm{T}}\right] \mathbf{T}+\left[\mathrm{A}_{\mathrm{q}}\right] \mathbf{q}+\left[\mathrm{A}_{\mathrm{M}}\right] \mathbf{M}=\mathbf{B}_{\text {external }} .
$$

Equations (1) and (2) can be solved thanks to a point matching approach. Thus, we get two new linear systems linking $\mathbf{T}, \mathbf{q}$, and M. By combining these two systems with system (3), we get a new linear system linking distribution of dipoles normal $\mathbf{T}$ to external field $\mathbf{B}_{\text {external }}$

$$
[\mathrm{A}] \mathbf{T}=\mathbf{B}_{\text {external }}
$$

where A matrix depends on the mesh, the material characteristics and the points where we want to compute the field (i.e., the sensors locations).

Equation (4) corresponds to a classical forward modeling. If we want to compute the field created by the machine, we have first to determine $\mathbf{T}$. For a healthy operation mode, its expression is known (flux in the air gap is a design parameter of the machine). It remains then to apply (4) to get the induction on sensors $\left(\mathbf{B}_{\text {external }}\right)$ with a simple matrix multiplication.

\section{INVERSE MODELING}

We now consider the inverse approach. From the external magnetic field measurements, the aim is to determine the magnetic induction in the machine air gap. So, we consider that $\mathbf{B}_{\text {external }}$ is known by measurement and $\mathbf{T}$ distribution has to be determined. We need then to solve the linear system (4).

Actually, the resolution of (4) cannot be so easy, because inverse problems can be classically ill-posed (matrix A can have a bad condition number). To overcome this problem, a good solution is to choose an optimal basis description for $\mathbf{T}$ distribution.

The induction in the machine air gap (i.e., the $\mathbf{T}$ distribution) can be represented with the following expression:

$$
\mathrm{T}(\theta, \mathrm{t})=\langle\mathrm{T}\rangle+\sum_{\mathrm{i}=1}^{\mathrm{p}} \mathrm{T}_{\mathrm{i}} \cos \left(\mathrm{i} \theta-\omega_{\mathrm{i}} \mathrm{t}\right) .
$$

This expression reminds us the classical expression of a rotating field in the air gap [4]. Note that $\langle\mathrm{T}\rangle$, which is the mean value of $\mathbf{T}$, is linked to the dissymmetry of ampere-turns of the rotor windings (further information will be given in Section IV).

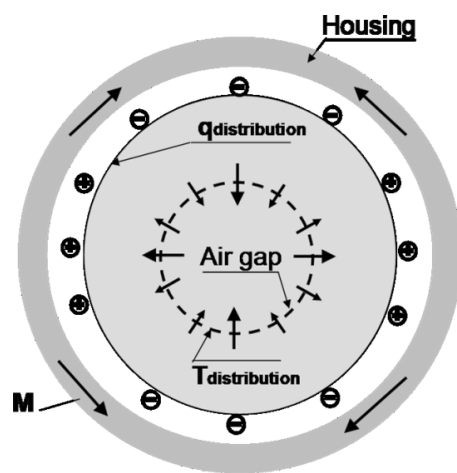

Fig. 1. Simplified model of a 4-pole electrical model with associated T, q, and M surface distributions; let us notice that the model is 3-D.

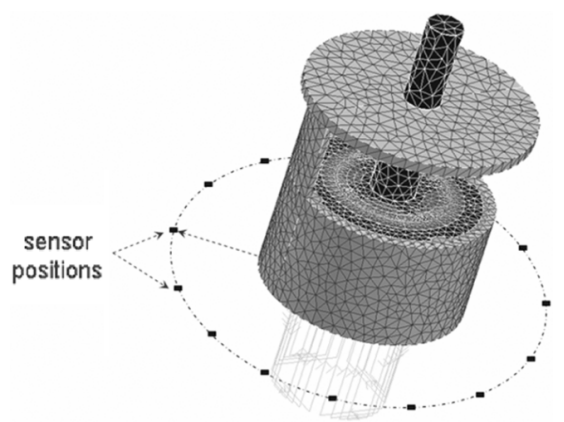

Fig. 2. 3-D homogenized FEM modeling of the machine with the magnetic sensor positions.

This basis description is very close to a Fourier expression and has the advantage to be orthogonal on the air gap surface. So, we can assume that it will be also the case in the space of radiated field. This property of orthogonality will then increase the robustness of the inversion process.

\section{Fault Detection on a Numerical Example}

\section{A. Numerical Measurements Generation}

Twelve tri-axis magnetic sensors have been regularly set around a 4-pole synchronous machine (see Fig. 2). In this section, the proposed approach has been only tested numerically (i.e., external magnetic field measurements are simulated by a FEM). In our application, the machine is an alternator with no load operation mode. We must notice that the presence of fault removes a part of symmetries of the problem. The 3-D modeling thus becomes very heavy. To simplify the mesh, a 3-D homogenized approach is used [6]. As a consequence, the whole machine is modeled with a healthy operation mode and a rotor short-circuit fault (18/162 loops). The different simulated "measurements" and thus different $\mathbf{B}_{\text {external }}$ can be obtained.

\section{B. Rotor Short Circuit Identification}

Let us consider the basis functions of $\mathbf{T}$ limited at the order $4\left(\langle\mathrm{~T}\rangle\right.$ and $\mathrm{T}_{\mathrm{i}}$ with $\left.\mathrm{i}=1,2,3,4\right)$. For symmetry reasons, only radial and orthoradial components are pertinent. We get then a linear system of 24 equations and 5 unknowns to determine. The basis description being orthogonal, (4) is solved thanks to pseudo-inversion based on a single value decomposition. 


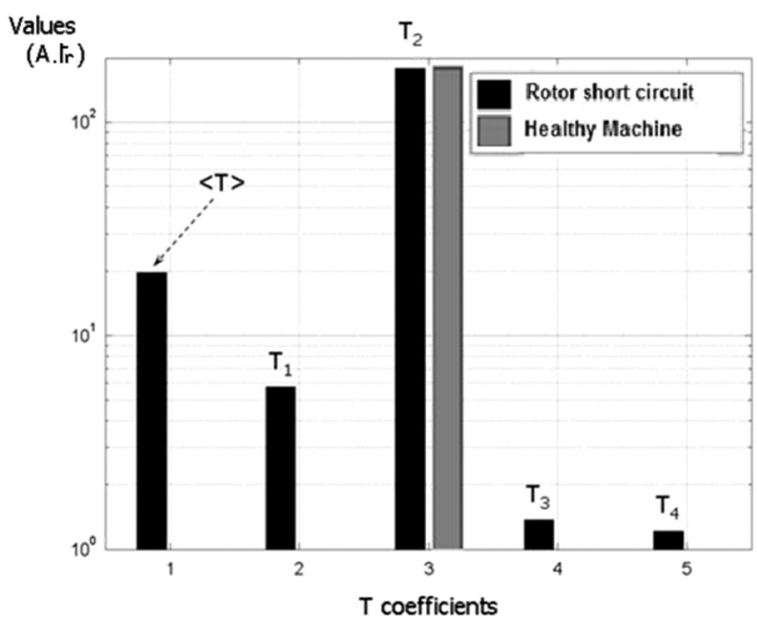

Fig. 3. Values of the first fifth $\mathbf{T}$ coefficients obtained by solving the inverse problems ( $\mathrm{p}$ is taken equal to 5 in (5))-healthy and rotor short-circuit cases.

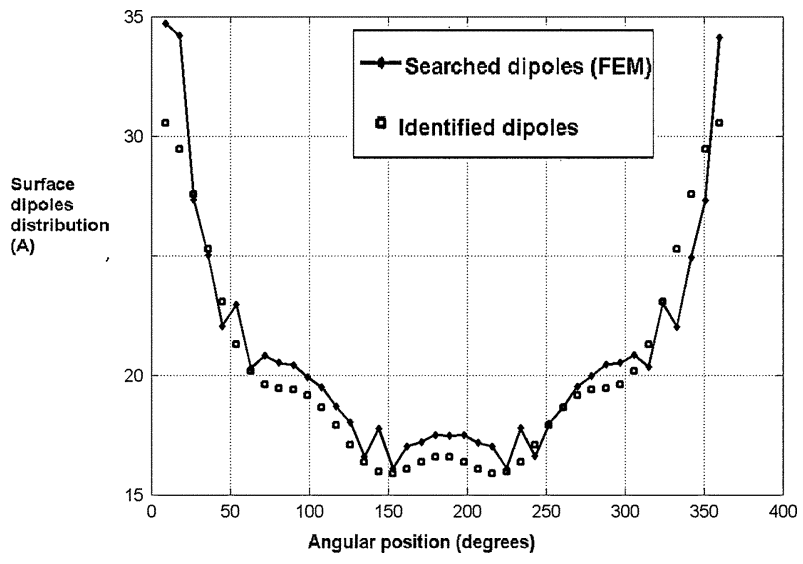

Fig. 4. Comparison between $\mathbf{T}$ distribution directly computed by FEM forward modeling and the $\mathbf{T}$ distribution identified thanks to inverse problem resolution. The curves represent the signature of the faults $\left(T_{\text {healthy }}-T_{\text {fault }}\right)$.

By solving (4) with the different measurement vectors obtained by FEM at a given time $t$, we get the following $\mathbf{T}$ distributions for each operation mode (healthy and rotor short circuit). Fig. 3 presents the coefficient obtained for both cases.

For the healthy operation mode, only one component appears $\left(\mathrm{T}_{2}\right)$. It corresponds to the 4 poles which are perfectly symmetric. For the short-circuit operation mode, new components appear $\left(\mathrm{T}_{1}, \mathrm{~T}_{3}\right.$, and $\left.\mathrm{T}_{4}\right)$ and the mean value $\langle\mathrm{T}\rangle$ is not equal to 0 . The appearance of these new components is typical of a fault signature. Thus, our approach allows us to detect it. We are going to see that it can also give an idea about its magnitude.

We now study more precisely the case of a rotor short circuit operation mode (the lost of about $10 \%$ of ampere-turns in one rotor pole). We have got a distribution of normal dipole $\mathbf{T}$ coefficients and then the $\mathrm{T}$ spatial distribution thanks to (5). This identified distribution can be compared with $\mathbf{T}$ distribution directly obtained thanks to our FEM forward model, by computing directly the induction in the air gap. For a better illustration, Fig. 4 presents the difference between $\mathbf{T}$ distribution obtained with the healthy operation mode and the short-circuit case.

Results present a really good agreement. We can conclude that our approach is reliable to reconstruct an accurate image
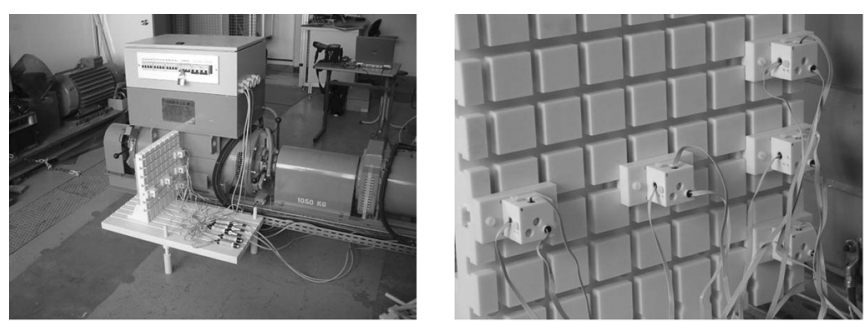

Fig. 5. Instrumentation of the machine with magnetic vector sensor system (fluxgate).

of the flux flowing in the air gap of the machine. Moreover, the identified curve can provide information about the importance of the fault. Let us come back to the definition of $\mathbf{T}$ distribution. In fact, $\mathbf{T}$ is the sum of $\mathbf{T}_{\mathbf{0}}$, representative of the rotor windings, and $\mathbf{T}_{\text {mat }}$, the reaction of the ferromagnetic material [1]. The divergence of the induction being free, $\left\langle\mathrm{T}_{\text {mat }}\right\rangle$ is always equal to zero. The mean value of $\mathbf{T}$ is then equal to the mean value of ampere-turn of the windings. If there is not any short-circuit fault on the rotor, $\left\langle\mathrm{T}_{0}\right\rangle$ is equal to zero like $\langle\mathrm{T}\rangle$. If now a short circuit appears, $\langle\mathrm{T}\rangle$ may help to quantify the number of short circuit turns.

Let us consider $\mathrm{S}_{\text {fault }}$ the surface of the short circuit coil and $\mathrm{n}_{\text {sc_turns }}$ the number of short-circuit turns. We get

$$
\mathrm{S}_{\text {fault }} \times \mathrm{n}_{\text {sc_turns }} \mathrm{I}_{\text {rotor }}=\mathrm{S}_{\text {airgap }} \times\langle\mathbf{T}\rangle \text {. }
$$

$\mathrm{I}_{\text {rotor }}$ being the current flowing in the rotor, $\mathrm{S}_{\text {airgap }}$ being the total surface of the air gap, and $\langle\mathrm{T}\rangle$ being identified by inverse problem we can evaluate the product

$$
\mathrm{S}_{\text {airgap }} \times\langle\mathrm{T}\rangle=2.51 \mathrm{~A} \cdot \mathrm{m}^{2}
$$

and compare it to

$$
\mathrm{S}_{\text {fault }} \times \mathrm{n}_{\text {sc_turns }} \mathrm{I}_{\text {rotor }}=2.67 \mathrm{~A} \cdot \mathrm{m}^{2}
$$

with $\mathrm{S}_{\text {airgap }}=0.13 \mathrm{~m}^{2}, \mathrm{~S}_{\text {fault }}=0.0078 \mathrm{~m}^{2}, \mathrm{n}_{\text {sc_turns }}=18$ and $\mathrm{I}_{\text {rotor }}=18 \mathrm{~A}$, and the identified $\langle\mathrm{T}\rangle$ value equals to $19.8 \mathrm{~A}$.

Both results are very similar (if we remember the number of assumptions done in our approach). It shows clearly the ability of the method to evaluate the importance of the fault. Let us notice that it is still difficult to discriminate several kinds of fault for which the product $\mathrm{S}_{\text {fault }} \times \mathrm{n}_{\text {sc_turns }}$ is the same. However, this discrimination seems to be possible by a shape analysis of the curve on Fig. 4. Moreover, let us notice that the fault localization can be simply carried out: a rotor position sensor makes it possible to set the $\mathrm{T}$ distribution in the rotor reference, the peak value corresponding to the short circuit coil axis. As a consequence, our approach allows us the localization and the quantification of a short-circuit fault which is a significant improvement in comparison with existing diagnosis approaches.

\section{FirSt EXPERIMENTAL VALIDATIONS OF FORWARD APPROACH}

A real mock-up of synchronous machine (alternator, $30 \mathrm{~kW}$, 4-poles) has been instrumented with a magnetic sensor system (fluxgates) coupled with a data acquisition chain (Fig. 5). This alternator allows to create rotor short circuits (18/162 loops on 


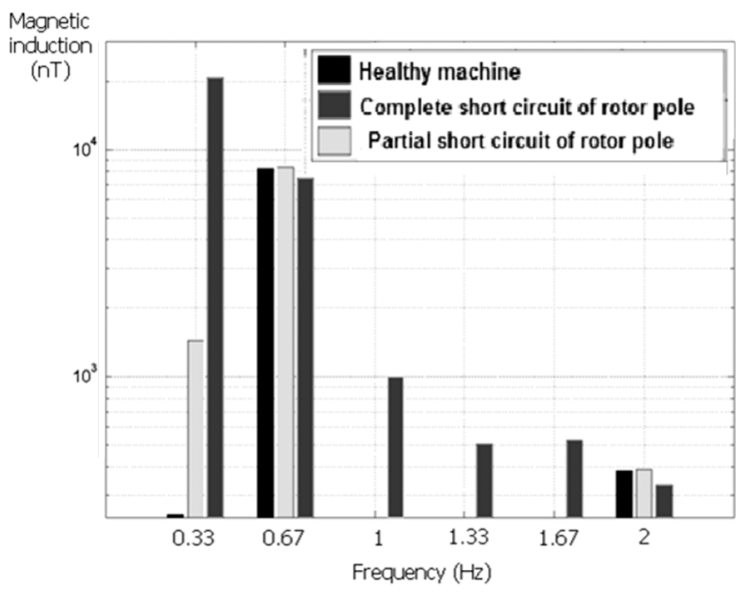

Fig. 6. Frequency spectrum of the flux leakage measured on one sensor. Comparison between healthy machine, partial rotor short-circuit, and complete pole short circuit.

one pole or one complete pole). The alternator excitation current has also been limited in order to operate within the linearity of materials. To limit the shielding effect of eddy currents, mainly located in the iron housing, we work at low rotation speed.

In a first step, the magnetic stray field is analyzed only on one sensor component. Fig. 6 shows the Fourier transform of the signal. For the healthy operation mode, a main frequency component appears at $0.67 \mathrm{~Hz}$ (corresponding to the pole rotation speed which is the double of the rotation speed-here $20 \mathrm{rd} / \mathrm{min}$ ). The additional components at the other frequencies (in particular at the rotation frequency $0.33 \mathrm{~Hz}$ ) are due to symmetry loss that occurs in case of rotor short-circuit fault. These specific spectrums are close to the one observed in the air gap: it is the image of the normal dipole distribution $\mathbf{T}$ obtained on Fig. 3.

Our new integral model has already been compared to finite element method and presents a good accuracy [1]. Our goal is now to validate our forward modeling with experimental data.

However, above our electrical machine a ferromagnetic case has been added: It includes electrical connectors that allow us to create rotor short circuit. In presence of this ferromagnetic body, the field values on the sensors are modified. We have first to quantify its influence.

The machine has been modeled by FEM with its ferromagnetic housing and the top case. Fig. 7 shows the modulus of field on three radial sensors at different distances from the housing. We can see that results of the computation present a very good adequacy with the measurements. Our forward modeling approach is then reliable. However, we can see that the influence of the top case cannot be neglected. This element must be added to our integral model as it has already been done for the iron housing. Moreover, the presence of the top case prevents us from putting our sensors regularly around the machine. So it seems difficult to apply our approach as it. Further work will deal with the application of the method with such sensors configuration.

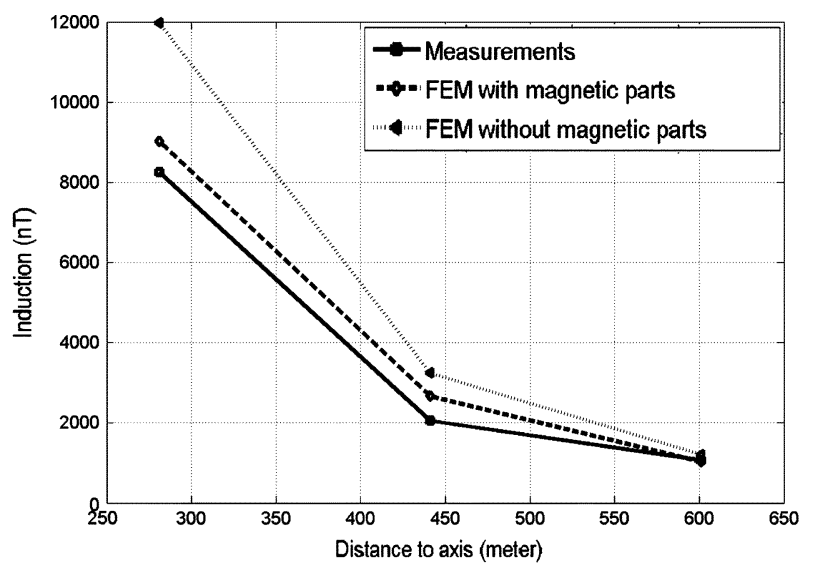

Fig. 7. Comparison of the field computation between FEM models and measurements (modulus of the magnetic field).

\section{CONCLUSION}

In this paper, we have proposed an original approach for the faults diagnosis of electrical machines. The approach consists in placing several magnetic sensors around the machine and analyzing the leakage flux. Moreover, we proposed to use inverse problem theory to allow the quantification of the fault and its localization which is a significant improvement of existing approaches. This study has been numerically validated and seems to be very promising. The forward modeling has been experimentally validated. In spite of the presence of a top case that strongly influences the stray field a good accordance has been found. Further works will deal with the application of inverse approach to experimental data.

\section{ACKNOWLEDGMENT}

This work was supported in part by the Institut National Polytechnique de Grenoble (INPG) and the Labs federation ELESA.

\section{REFERENCES}

[1] O. Chadebec, V.-P. Bui, L.-L. Rouve, and J.-L. Coulomb, "A fast model to predict the stray magnetic field created by electrical machines," in Proc. CEFC 2006, Miami, FL, May 2006.

[2] O. Mun Kwon, C. Surussavadee, M. V. K. Chari, and S. Salon, "Analysis of the far field of permanent-magnet motors and effects of geometric asymmetries and unbalance in magnet design," IEEE Trans. Magn., vol. 40, no. 2, pp. 435-442, Mar. 2004.

[3] B. Froidurot, L.-L. Rouve, A. Foggia, J.-P. Bongiraud, and G. Meunier, "Magnetic discretion of naval propulsion machines," IEEE Trans. Magn., vol. 38, no. 2, pp. 1185-1188, Mar. 2002.

[4] P. L. Timar, Noise and Vibration of Electrical Machines. New York: Elsevier, 1989.

[5] O. Chadebec, J.-L. Coulomb, and F. Janet, "A review of magnetostatic moment method," IEEE Trans. Magn., vol. 42, no. 4, pp. 515-520, Apr. 2006.

[6] V.-P. Bui, O. Chadebec, L.-L. Rouve, and J.-L. Coulomb, "A homogenized FEM model to predict the stray magnetic field created by faulty electrical machines," in Proc. CEFC 2006, Miami, FL, May 2006.

Manuscript received June 24, 2006. Corresponding author: O. Chadebec (e-mail: olivier.chadebec@g2elab.inpg.fr). 